\section{Neher, Erwin}

\section{W. Hubl}

Ehem. Krankenhaus Dresden-Friedrichstadt, Institut für Klinische Chemie und Labormedizin, Dresden, Deutschland

Lebensdaten Deutscher Biophysiker, geboren am 20. März 1944 in Landsberg am Lech. Bereits im Gymnasium interessierte sich Neher für Kybernetik, Fragen des Nervensystems und Biophysik. Von 1963-1966 Physikstudium an der TU München und ab 1966 an der University of Wisconsin (Madison). Im Jahr 1970 promovierte er am Max-PlanckInstitut für Psychiatrie in München. Seit 1972 ist Neher am Max-Planck-Institut für biophysikalische Chemie in Göttingen tätig. Von 1975-1976 arbeitete er als Gastwissenschaftler an der Yale University am Department of Physiology in New Haven in Connecticut. Seit 1983 ist Neher Direktor am MaxPlanck-Institut für biophysikalische Chemie in Göttingen und Leiter der Abteilung Membranbiophysik. Im Jahr 1989 war er als Fairchild Scholar am Californian Institute of Technology tätig. Das gegenwärtige Interesse von Erwin Neher konzentriert sich auf die Auslösung der Hormon- und Neurotransmitterfreisetzung durch Calcium und calciumspezifische Ionenkanäle. Er ist seit 2002 DFG-Teilprojektleiter „Quantitative Molecular Microscopy“ im Projekt zur Molekularphysiologie des Gehirns.
Verdienste Ab 1976 arbeitete Neher mit Bert Sakmann an der Universität Göttingen an der Entwicklung der PatchClamp-Technik, welche die Grundlagen für Entdeckungen zur Funktion der Ionenkanäle bildeten. Hierbei gelang es den Forschern, geringe elektrische Ströme zwischen Körperzellen und der Umgebung zu registrieren und damit erstmals den Nachweis zu erbringen, dass in der Zellhülle winzige Kanäle existieren, die den Durchfluss von Ionen (Kalium, Natrium) vom Zellinnern in die Umgebung ermöglichen. Dadurch war es möglich geworden, zahlreiche Erkrankungen mit einer defekten Regulierung des Ionenflusses aufzuklären. Im Jahr 1991 erhielt Neher gemeinsam mit Sakmann den Nobelpreis für Physiologie und Medizin für die Entdeckungen zur Funktion von einzelnen Ionenkanälen in den Zellen. Erwin Neher erhielt ferner eine Ehrenprofessur in Göttingen, 10 Ehrendoktortitel auf 4 Kontinenten, im Jahr 1987 den Gottfried-Wilhelm-Leibniz-Preis der Deutschen Forschungsgemeinschaft, im Jahr 1990 den Niedersächsischen Staatspreis für Wissenschaft und den Bristol-Myers Squibb Research Award, New York sowie ein Jahr später den Gerard Prize, der American Neuroscience Association.

\section{Literatur}

Frängsmyr T (1992) Les Prix Nobel. The Nobel Prizes 1991. Nobel Foundation, Stockholm

Neher E (2015) Merits and limitations of vesicle pool models in view of heterogeneous populations of synaptic vesicles. Neuron 87:1131-1142 